\title{
Validez y confiabilidad del instrumento de calidad de vida de Betty Ferrell en español, para personas con enfermedad crónica'
}

Lina Rocío Corredor Parra²

Gloria Mabel Carrillo³

doi:10.11144/Javeriana.ie18-1.vcic

Como citar: Corredor Parra LR, Carrillo GM. Validez y confiabilidad del instrumento de calidad de vida de Betty Ferrell en español, para personas con enfermedad crónica. Investig Enferm. Imagen Desarr. 2016;18(1):129-148. http://dx.doi.org/10.11144/ Javeriana.ie18-1.vcic

1. Artículo original de investigación. Recibido: 25 de mayo de 2015. Aprobado: 18 de septiembre de 2015.

2. Enfermera. Magíster en Enfermeria con énfasis en Cuidado al Paciente Crónico, Universidad Nacional de Colombia. Correo electrónico: 1rcorredorp@unal.edu.co

3. Enfermera. Magíster en Enfermería. Profesora asociada, Universidad Nacional de Colombia. Correo electrónico: gmcarrillog@unal.edu.co 


\section{Resumen}

Los instrumentos utilizados para evaluar la calidad de vida deben suministrar información real, segura y adecuada. Por ello deben seguir procesos psicométricos de validez y confiabilidad que permitan su uso en el contexto nacional y latinoamericano y evidenciar que miden cuanto pretenden medir y con un alto grado de precisión. Objetivo: Determinar la validez y confiabilidad del instrumento calidad de vida de Betty Ferrell, para personas con enfermedad crónica, en Colombia. Método: Estudio de abordaje cuantitativo descriptivo de corte transversal metodológico. Participaron 287 personas con enfermedad crónica no transmisible. Se realizó validez de contenido por medio del índice de concordancia de kappa y el índice de validez de contenido y se realiza validez de constructo, así como confiabilidad por consistencia interna mediante el alfa de Cronbach. Resultados: Se determinó una validez de contenido adecuada con un índice de validez de contenido (IVC) de 0,9, e índice de kappa de 0,61. La consistencia interna por medio del alfa de Cronbach fue de 0,88 . Respecto a la validez de constructo, al análisis factorial exploratorio solo 22 de los 41 items se explican por el factor subyacente a la dimensión planteada por el autor y se observa discrepancia en las dimensiones de bienestar psicológico y bienestar social. Conclusiones: El instrumento de calidad de vida de Betty Ferrell para personas con enfermedad crónica es confiable, pues tiene un adecuado índice de validez de contenido para su uso en el contexto local. Se requiere seguir estudiando el constructo, probablemente con la eliminación de ítems y análisis factorial confirmatorio.

Palabras clave: calidad de vida; enfermedad crónica; psicometría; validez de las pruebas

\section{Validity and Reliability of the Instrument of Quality of Life of Betty Ferrell in Spanish, for People with Chronic Disease}

\section{Abstract}

The instruments used to assess quality of life are useful tools that should provide real, safe and adequate information to promote programs, diagnose situations, to evaluate treatments, among others. Therefore must follow psychometric validity and reliability processes that allow its use in the local context, showing that measures what it purports to measure with a high degree of accuracy. Objective: To determine the validity and reliability of the Betty Ferrell's quality of life instrument, for people with Chronic disease, for Colombian context, by conducting psychometric tests. Methods: Descriptive study of cross-sectional quantitative approach. It was conducted; facial, content and construct validity was made, reliability for internal consistency using Cronbach's alpha. Results: The instrument adequate content validity with 0.9 , internal consistency using Cronbach's alpha was 0.88, it was adequate. Regarding construct validity: factorial analysis only 22 of the 41 items are explained by the underlying dimension factor raised by the author, showing marked discrepancy in the dimensions of Psychological and Social Welfare. Conclusions: The quality of life instrument Betty Ferrell for people with chronic disease requires further study construct validity, it is recommended to delete items and perform a confirmatory factor analysis.

Keywords: quality of life; chronic illness; psychometrics; validity of tes 


\section{Validade e fiabilidade do instrumento de qualidade de vida da Betty Ferrell em espanhol, para pessoas com doença crônica}

\section{Resumo}

Os instrumentos utilizados para avaliar a qualidade de vida são ferramentas úteis que deve fornecer informação real, segura e adequada. Por que eles devem seguir os processos de validade e confiabilidade psicométricas que permitem a sua utilização no contexto da América Latina nacional e mostrando que medida o que pretende medir e com um alto grau de precisão. Para determinar a validade e confiabilidade do instrumento de qualidade de vida de Betty Ferrell, para as pessoas com doenças crônicas na Colômbia. Método: Estudo de abordagem metodológica descritivo transversal quantitativo. Eles envolveu 287 pessoas com doenças crônicas não transmissiveis. validade de conteúdo por meio do índice de índice de concordância e validade de conteúdo foi realizada kappa e a validade de construto e confiabilidade é de consistência interna por alfa de Cronbach. Resultados: Válidos para conteúdo apropriado com o índice de validade de conteúdo (IVC) de 0,9 e índice de kappa de 0,61, a consistência interna por alfa de Cronbach foi de 0,88 foi determinada. Quanto à validade de constructo, análise fatorial exploratória com apenas 22 dos 41 itens são explicados pelo factor dimensão subjacente levantada pelo autor observou discrepância no tamanho de Psicológica e da Previdência Social. Conclusões: O instrumento de qualidade de vida de Betty Ferrell para pessoas com doença crônica é de confiança porque tem um índice de validade de conteúdo adequado para utilização no contexto local. Ele requer um estudo mais aprofundado a construção, provavelmente removendo itens e análise fatorial confirmatória.

Palavras chave: qualidade de vida; doença crônica psicometria; validade dos testes 


\section{Introducción}

Las enfermedades no transmisibles (ENT), también llamadas enfermedades crónicas, son la principal causa de mortalidad en el mundo. Los datos disponibles demuestran que casi el $90 \%$ de las muertes por ENT se dan en los países de ingresos medios y bajos, aparte de que tienen un efecto nocivo en la vida humana y la economía de los países. Estas enfermedades reducen la calidad de vida de los pacientes, provocan muerte prematura y dan lugar a otras consecuencias adversas $(1,2)$.

La calidad de vida de las personas con enfermedad crónica es un fenómeno de interés investigativo que se debe explorar desde diferentes perspectivas: desde el abordaje de las dimensiones física, social, psicológica y espiritual que la constituyen; desde la percepción de los pacientes, y desde la identificación, construcción y estandarización de instrumentos de medición que permitan reportar la realidad percibida por las personas.

La calidad de vida se ha evaluado desde diferentes enfoques: desde el punto de vista demográfico, la calidad de vida se ve como la satisfacción de necesidades básicas en términos físicos de vivienda y servicios públicos; desde el componente social, se comprende a partir del componente de salud, cultura, educación, recreación y organización comunitaria, y desde la perspectiva en salud, se percibe a partir de las dimensiones físicas, psicológicas, emocionales, interacción somática y funcionamiento familiar.

Existen diferentes conceptualizaciones de la calidad de vida relacionadas con salud. La Organización Mundial de la Salud (3) la define como "la percepción del individuo de su posición en la vida en el contexto de la cultura y sistemas de valores en los que vive y en relación con sus objetivos, expectativas, valores e intereses". Desde el enfoque psicológico, Oblitas (4) la define como una evaluación objetiva y subjetiva de, al menos, los siguientes elementos: salud, educación, trabajo, vivienda, seguridad social, vestido, ocio y derechos humanos; además puede ser definida, en términos generales, como una medida compuesta de bienestar físico, mental y psicológico, tal como lo percibe cada persona y grupo. Desde el punto de vista de enfermería, Ferrell (5) la describe como una construcción multidimensional que incluye el bienestar o descontento en aspectos de la vida importante para el individuo, que abarca la interacción de la salud y el funcionamiento psicológico, espiritual, socioeconómico y familiar.

Evaluar la calidad de vida de personas con enfermedad crónica no transmisible es importante, porque con base en datos confiables se puede conocer el impacto de la enfermedad o tratamiento y la adaptación de la persona a la enfermedad, evaluar las diferentes terapias, facilitar la rehabilitación de los pacientes, al igual que implementar acciones que mejoren la calidad de vida de estas personas.

Los instrumentos utilizados para evaluar la calidad de vida son herramientas útiles que suministran información real, segura y adecuada para promover programas, diagnosticar situaciones, evaluar tratamientos, siempre y cuando se les hayan realizado las pruebas psicométricas de validez 132 y confiabilidad (6,7). Ferrell y cols. (5) diseñaron un instrumento para medir la calidad de vida, versión paciente y versión cuidador familiar, el cual 
inicialmente se orientó a pacientes sobrevivientes de cáncer, producto de varias investigaciones cualitativas realizadas.

Una vez consolidada teóricamente, la escala se sometió a diferentes pruebas de validez y confiabilidad:

1. Estabilidad por medio de test retest con un resultado de 0,89. En las subescala física: 0,88 , en la psicológica: 0,88 , en la social: 0,81 y en la espiritual 0,90 .

2. Consistencia interna usando el alfa de Cronbach con hallazgos de 0,93 en general. En las subescalas física: 0,81, psicológica: 0,89, social: 0,81 y espiritual: 0,71 .

3. Validez de contenido con investigadores y enfermeros con experiencia en oncología.

4. Regresión múltiple por pasos para determinar los factores más predictivos del instrumento, con lo cual se encontraron 17 variables estadísticamente significativas para explicar el $91 \%$ de la varianza global del instrumento.

5. Correlaciones de Pearson para estimar las relaciones entre las subescalas del instrumento de calidad de vida sobrevivientes del cáncer y las subescalas del instrumento FACT-G. Asî se obtuvo una correlación de moderada a fuerte entre las subescalas de la dimensión física del Instrumento de Calidad de Vida Sobreviviente del Cáncer (CDVSC) y la física del instrumento FACT, que fue de 0,74. Entre la psicológica de CDVSC y la emocional del FACT fue de 0,65; la de bienestar social de CDVSC y la social del FACT fue de 0,78.

Medidas de validez adicionales incluyeron la correlación individual entre items del instrumento CDVSC, análisis factorial y validez de constructo discriminante. Los resultados demostraron que el instrumento de CDVSC y sus subescalas miden adecuadamente la calidad de vida de la creciente población sobreviviente del cáncer (5).

El Grupo de Cuidado a la Persona con Enfermedad Crónica y la Familia de la Universidad Nacional de Colombia, con la autorización de los autores, realizó una retraducción al español, adaptación a escala Likert y la estandarización para personas con enfermedades crónicas, teniendo en cuenta que las dimensiones de esta escala son similares para personas con enfermedades crónicas, incluido el cáncer.

Con el fin de que este instrumento se pueda aplicar en el contexto local, se requiere completar el proceso psicométrico de validez y confiabilidad en español; de esta forma, se tendrá certeza de contar con una escala que brinde información acertada sobre la calidad de vida de los pacientes con enfermedad crónica en Colombia. Por lo tanto, el objetivo de esta investigación fue determinar la validez de contenido, validez de constructo y confiabilidad del Instrumento de Calidad de Vida de Betty Ferrell para personas con enfermedad crónica.

\section{Método}

Estudio metodológico de abordaje cuantitativo y de corte transversal que constó de dos fases para alcanzar el objetivo propuesto: 
1. Validez facial y contenido: se conformó un grupo de 9 expertos con tîtulos de especialización, maestría y doctorado en enfermería y áreas afines, que tenían entre 10 y 40 años de experiencia, con el fin de determinar si el instrumento realmente medía lo que se proponía medir. Estos expertos evaluaron la validez de contenido de acuerdo con lo propuesto por Lawshe (8): revisión de ítems en su coherencia, suficiencia, relevancia y esencia (9). Para determinar el grado de acuerdo entre jueces, respecto a las categorías evaluadas en la validez facial y de contenido, se utilizó el índice de concordancia kappa de Fleiss (10), que se consideró aceptable, cuando los índices kappa sean mayores de 0,61 , lo cual representa una fuerza de concordancia entre expertos, buena y muy buena. En esta fase se diseñó un formato teniendo en cuenta las recomendaciones dadas por Escobar y Cuervo (11).

2. Validez de constructo y confiabilidad del instrumento: los datos se recolectaron aplicando el instrumento de calidad de vida de Betty Ferrell, para personas con enfermedad crónica, a usuarios con enfermedad crónica no transmisible. El tamaño de la muestra requerido para determinar la validez de constructo y confiabilidad se obtuvo de acuerdo con el número de ítems del instrumento. En esta investigación se determinaron como participantes 7 personas por item del instrumento (12-14); por lo tanto, la muestra fue de 287 personas con enfermedad crónica.

Los criterios de inclusión que se tuvieron en cuenta fueron los siguientes: personas mayores de 18 años que supieran leer y escribir, personas con enfermedad crónica no transmisible como: hipertensión arterial (HTA), insuficiencia renal crónica (IRC), enfermedad pulmonar obstructiva crónica (EPOC) y cáncer (CA) de cualquier etiología. Los criterios de exclusión fueron: personas con alguna alteración mental, personas que se encontraran bajo el efecto o uso habitual de sustancias psicoactivas.

Se realizó inmersión en el campo por cuatro meses en los servicios de hospitalización de la Clínica Universitaria Colombia, con captación diaria de participantes, verificación de cumplimiento de criterios de inclusión y se realizó un acercamiento al paciente explicándole en qué consistían las preguntas que se iban a hacer y se firmó el consentimiento informado.

Los datos de caracterización de las personas con enfermedad crónica se obtuvieron por medio del instrumento de caracterización del paciente con enfermedad crónica GCPC-UN-P (15), del Grupo de Cuidado al Paciente Crónico y su Familia de la Universidad Nacional de Colombia.

La validez de constructo se realizó por medio de la técnica de análisis factorial exploratorio utilizando el método de componentes principales y rotación Varimax. Este trabajo se acogió a la opinión presentada por Nunally y Bernestein (7), quienes consideran cuatro criterios en la evaluación de los items del instrumento: 1) presencia de una carga factorial (loading) igual o superior a 0,3 con comunalidad al menos de 0,$1 ; 2)$ ausencia de efecto adverso sobre las medidas de confiabilidad calculadas y visualizadas en la curva de Cronbach-Mesbah (16), y 3) ausencia de correlación fuerte (superior a 0,8) 
y significancia con los demás ítems. La confiabilidad se realizó por medio de consistencia interna con el alfa de Cronbach.

En los aspectos éticos se tuvo en cuenta la Resolución 8430 de 1993 del Ministerio de Salud de Colombia (17), la Ley 911 de 2004 (18) y las pautas éticas internacionales para la investigación biomédica con seres humanos (19), además de la aprobación del Comité de Ética de la Facultad de Enfermería de la Universidad Nacional de Colombia y de la Fundación Universitaria Sanitas. Cumplió con la politica ambiental de la Universidad Nacional de Colombia sobre el uso adecuado y responsable de los recursos (20). La autora del instrumento autorizó realizar los ajustes necesarios para poder aplicar el instrumento en el contexto colombiano.

Los hallazgos reportados fueron digitados en una base de datos de Excel y posteriormente exportados al programa SPSS versión 19.0, bajo licencia de la Universidad Nacional de Colombia.

\section{Resultados}

\section{Fase 1. Validez de contenido del Instrumento de Calidad de Vida de Betty Ferrell, para personas con enfermedad crónica}

Índice de kappa de Fleiss. La validez de contenido estuvo determinada por la suficiencia, la coherencia y la relevancia de la versión modificada con readecuación semántica del Instrumento de Calidad de Vida de Betty Ferrell, para personas con enfermedad crónica. Los resultados muestran una fuerza de concordancia buena entre jueces, lo que concluye que los expertos estuvieron de acuerdo en afirmar que el instrumento es suficiente, coherente y relevante para medir la calidad de vida de las personas con enfermedad crónica (tabla 1).

TABla 1. Resultados del índice de kappa de Fleiss: suficiencia, coherencia y relevancia

\begin{tabular}{|l|c|c|c|}
\hline & Suficiencia & Coherencia & Relevancia \\
\hline $\mathrm{K}=$ & 0,71 & 0,68 & 0,71 \\
\hline
\end{tabular}

Fuente: elaboración propia

Índice de contenido. El análisis y la interpretación se basó en el modelo de Lawshe modificado (8), el cual representa una propuesta de comprobación útil para la valoración de la validez de contenido de las pruebas diseñadas, adaptadas o utilizadas en enfermería. Para cada ítem se calculó la razón de validez de contenido modificada (CVR') y para todo el instrumento se obtuvo el índice de validez de contenido (CVI).

Como se observa en la tabla 2 , los resultados evidenciaron que el CVI para el instrumento en su medición global fue de 0,9, lo cual indica que la validez de contenido del instrumento es adecuada; sin embargo, los items que obtuvieron un índice menor de 0,58 deben revisarse nuevamente. Llama la atención que la mayoría de ítems están concentrados en la dimensión de bienestar psicológico, y adjudicando a los ítems de bienestar social y espiritual esencialidad en su totalidad. 


\begin{tabular}{|c|c|c|c|c|c|c|c|c|c|c|c|c|c|c|c|c|c|c|c|}
\hline & $\begin{array}{l}\hat{A} \\
\text { o }\end{array}$ & \begin{tabular}{|l|}
10 \\
10 \\
0 \\
0
\end{tabular} & $\begin{array}{l}8 \\
\circ \\
-i\end{array}$ & $\begin{array}{l}\hat{A} \\
\hat{0}\end{array}$ & $\begin{array}{l}m \\
0 \\
0 \\
0\end{array}$ & $\begin{array}{l}\hat{N} \\
0\end{array}$ & {$\left[\begin{array}{l}10 \\
10 \\
0\end{array}\right.$} & $\begin{array}{l}\hat{N} \\
0^{\prime}\end{array}$ & & $\begin{array}{l}\hat{N} \\
\hat{0}\end{array}$ & $\begin{array}{l}\Rightarrow \\
0\end{array}$ & $\begin{array}{l}m \\
\text { m } \\
0\end{array}$ & $\Rightarrow$ & $\begin{array}{l}m \\
m \\
0 \\
0\end{array}$ & \begin{tabular}{|l|}
2 \\
1 \\
0 \\
0
\end{tabular} & $\begin{array}{l}10 \\
10 \\
0 \\
0\end{array}$ & $\begin{array}{l}7 \\
0 \\
0\end{array}$ & $\begin{array}{l}10 \\
10 \\
0 \\
0\end{array}$ & $\stackrel{8}{8}$ \\
\hline & - & $a$ & 0 & - & $\infty$ & -1 & $a$ & -1 & & -1 & 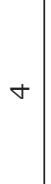 & $m$ & + & $m$ & $a$ & $a$ & $\theta$ & $a$ & 0 \\
\hline 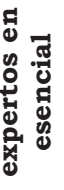 & $\infty$ & $\Lambda$ & $a$ & $\infty$ & 0 & $\infty$ & $\wedge$ & $\infty$ & & $\infty 1$ & in & 0 & 0 & 0 & 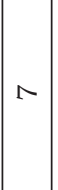 & $\wedge$ & in & $\Lambda$ & $a$ \\
\hline 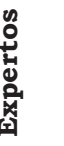 & $a$ & $a$ & $a$ & $a$ & $a$ & $a$ & $a$ & $a$ & & $a / b+3$ & $a$ & $a$ & $a$ & $a$ & $a$ & $a$ & $a$ & $a$ & $a$ \\
\hline ⿷匚ّ & 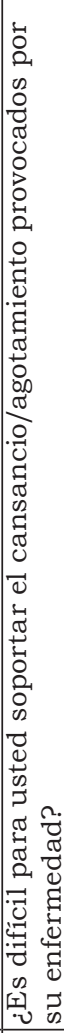 & 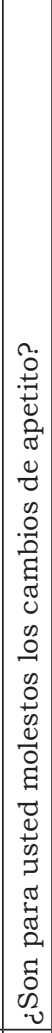 & 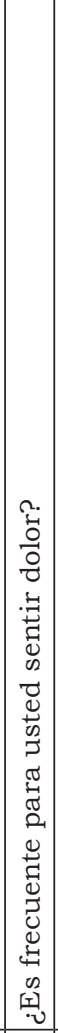 & 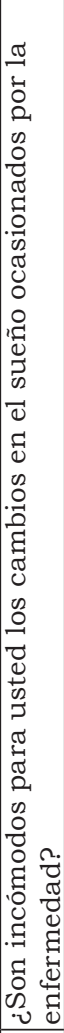 & 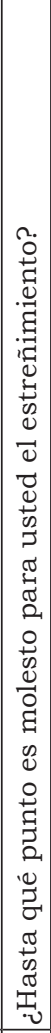 & 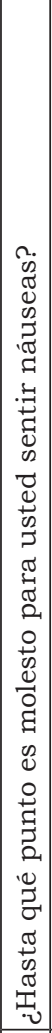 & 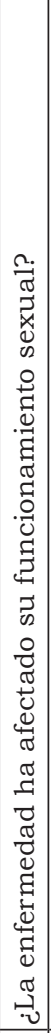 & 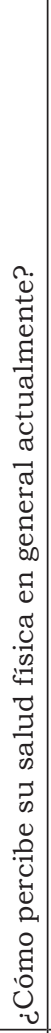 & 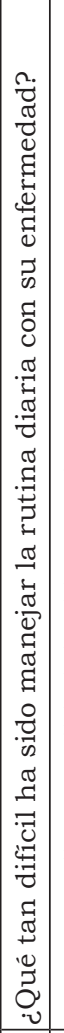 & 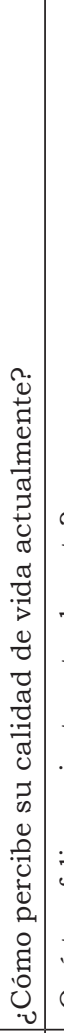 & 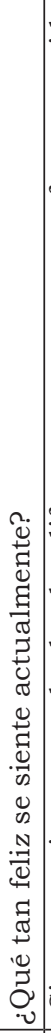 & 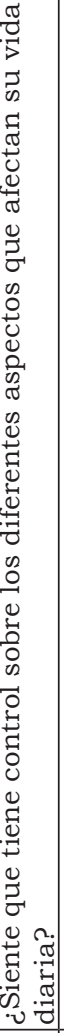 & 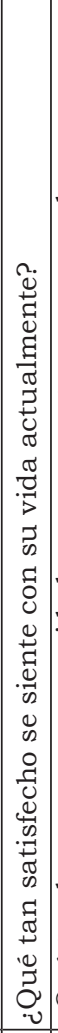 & 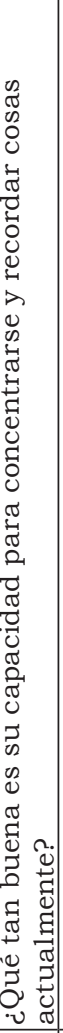 & 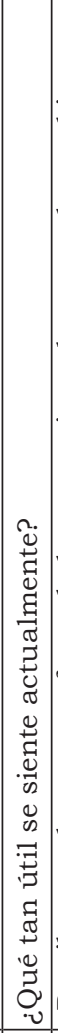 & 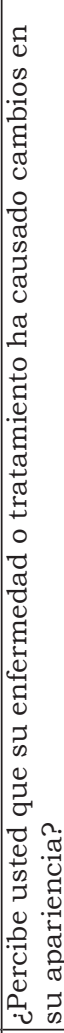 & 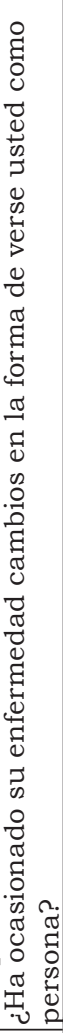 & 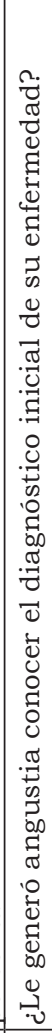 & 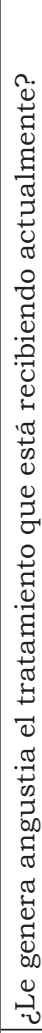 \\
\hline$z$ & $\neg$ & $\mathrm{N}$ & $\infty$ & $\nabla$ & in & 0 & $\wedge$ & $\infty$ & $a$ & $\stackrel{ }{\circ}$ & $\Rightarrow$ & $\stackrel{N}{\sim}$ & $\stackrel{m}{-}$ & $\underset{\nabla}{ \pm}$ & $\stackrel{20}{\rightarrow}$ & $\stackrel{\bullet}{-}$ & 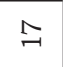 & $\stackrel{\infty}{\rightarrow}$ & $\stackrel{2}{2}$ \\
\hline & & & O!St & f Uọ! & suət & w! & & & \multicolumn{11}{|c|}{ 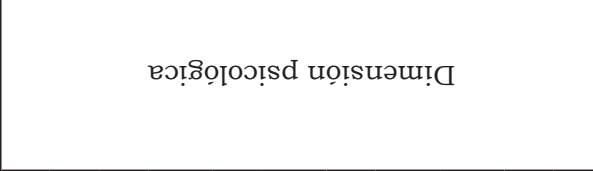 } \\
\hline
\end{tabular}




\begin{tabular}{|c|c|c|c|c|c|c|c|c|c|c|c|c|c|c|c|}
\hline ơ & $=$ & $\mid \begin{array}{l}1 \\
10 \\
0 \\
0\end{array}$ & $\underset{-}{\stackrel{0}{-}}$ & 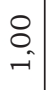 & $\begin{array}{l}\hat{A} \\
0 \\
0\end{array}$ & $\begin{array}{c}\hat{A} \\
0^{\prime}\end{array}$ & $\begin{array}{l}D_{0} \\
0 \\
0\end{array}$ & $\mid \begin{array}{l}8 \\
-i\end{array}$ & 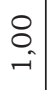 & $\begin{array}{l}\hat{A} \\
\hat{0}\end{array}$ & $\begin{array}{l}\hat{A} \\
0 \\
0\end{array} \mid$ & 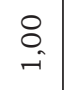 & $\underset{-}{\stackrel{-}{-}}$ & $\underset{-}{8}$ & $\stackrel{-}{-1}$ \\
\hline 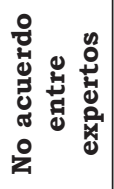 & t & $a$ & 0 & 0 & -1 & $\neg$ & $\mathrm{c}$ & 0 & 0 & -1 & -1 & 0 & 0 & 0 & 0 \\
\hline 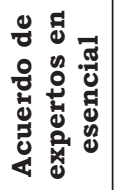 & 10 & $\Lambda$ & $a$ & $a$ & $\infty$ & $\infty$ & $\wedge$ & $a$ & $a$ & $\infty$ & $\infty$ & $a$ & $a$ & $a$ & $a$ \\
\hline 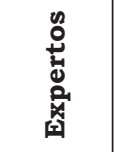 & $a$ & $a$ & $a$ & $a$ & $a$ & $a$ & $a$ & $a$ & $a$ & $a$ & $a$ & $a$ & $a$ & $a$ & $a$ \\
\hline$\underset{\varpi}{\mathbb{\Xi}}$ & 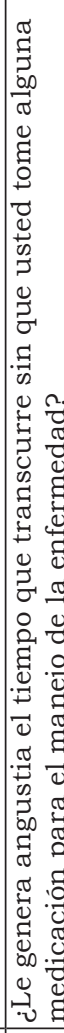 & 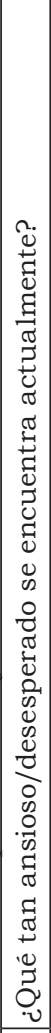 & 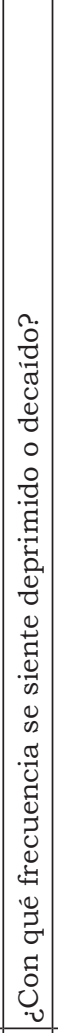 & 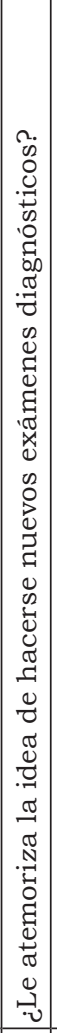 & 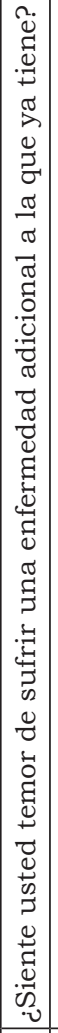 & 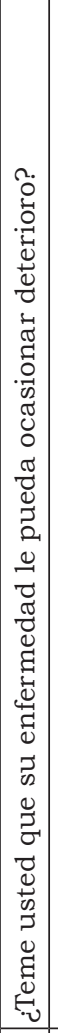 & 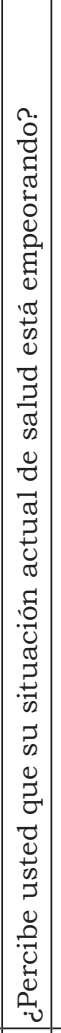 & 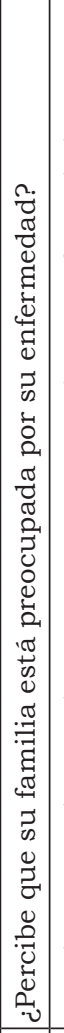 & 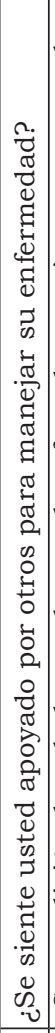 & 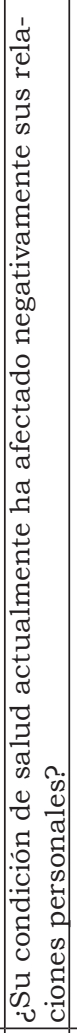 & 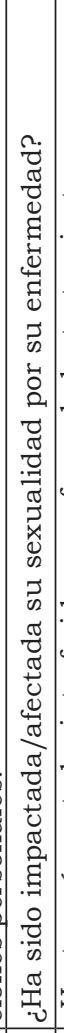 & 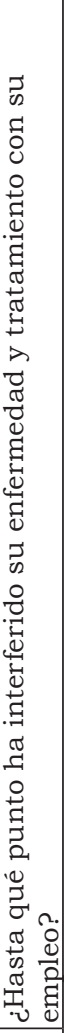 & 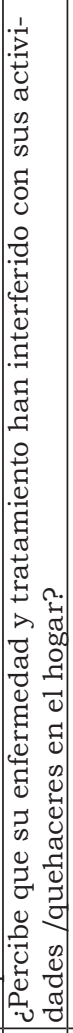 & 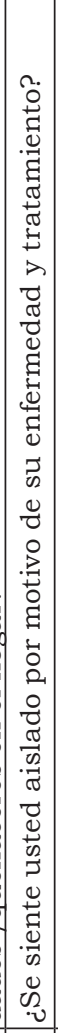 & 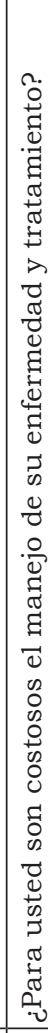 \\
\hline ì & $\stackrel{\curvearrowright}{\sim}$ & $\overrightarrow{\mathrm{v}}$ & $\stackrel{N}{N}$ & $\stackrel{m}{\mathrm{~N}}$ & $\stackrel{\sim}{\sim}$ & $\stackrel{\stackrel{\sim}{n}}{\sim}$ & $\stackrel{\bullet}{\sim}$ & $\hat{\mathrm{v}}$ & $\stackrel{\infty}{\sim}$ & ते & ○ & $\vec{m}$ & ले & $\begin{array}{ll}m \\
m\end{array}$ & ले \\
\hline & \multicolumn{9}{|c|}{ вว!̣̊ōoo!̣sd uọ̣suəu!̣ } & \multicolumn{6}{|c|}{ [е!̣oos uộsuəu!̣ } \\
\hline
\end{tabular}




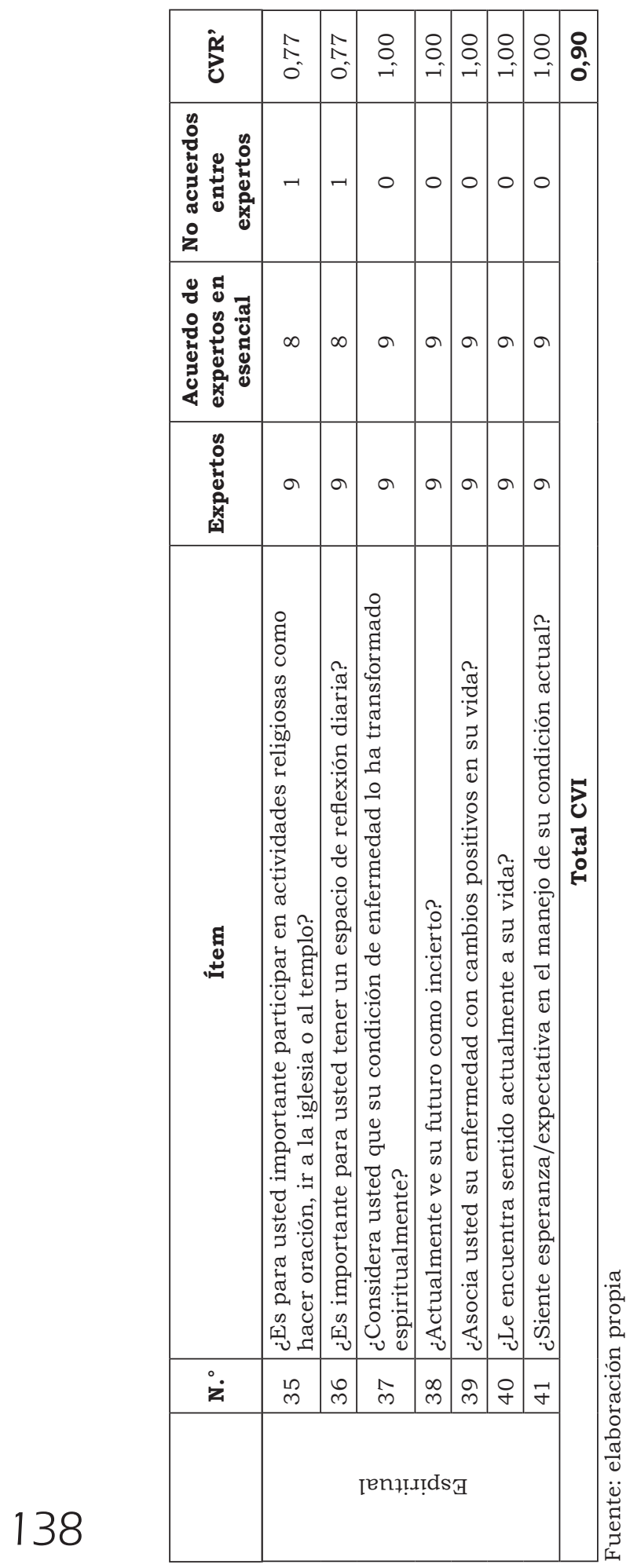




\section{Fase 2. Validez de constructo y confiabilidad del Instrumento de Calidad de Vida de Betty Ferrell, para personas con enfermedad crónica}

Validez de constructo. De acuerdo con lo referido en el método, se identificó el cumplimiento de tres condiciones necesarias para realizar el análisis factorial. Se obtuvo una muestra de personas con enfermedad crónica mayor de 100 ( $\mathrm{n}=287$ participantes); un índice de medida de adecuación de la muestra (KMO), si $\mathrm{KMO} \geq 0,9$, el test es muy bueno; notable para $\mathrm{KMO} \geq$ 0,8 ; mediano para $\mathrm{KMO} \geq 0,7$; bajo para $\mathrm{KMO} \geq 0,6$, y muy bajo para $\mathrm{KMO}<$ 0,5. El KMO obtenido fue de 0,865; prueba de esfericidad de Barlett, si Sig < 0,05 se puede aplicar el análisis factorial; si Sig > 0,05 no se puede aplicar el análisis factorial, que para el estudio fue de 0,00.

\section{Análisis factorial}

En este estudio se tomaron los criterios presentados por Raykov y Marcoulides (21) y Waltz, Strikland y Lenz (22), según los cuales se consideran no significativos los pesos factoriales inferiores a 0,3 o los valores de comunalidad inferiores a 0,1 .

En la tabla 3 se presenta el resultado para el análisis factorial exploratorio ejecutado. Inicialmente, se extrajeron 5 factores; sin embargo, el quinto factor no proporcionó mayor información, por lo que se dejaron 4 factores. El análisis del efecto de los ítems en la validez de constructo del instrumento, evaluada con el valor de la carga factorial del item sobre el factor y con el valor de la comunalidad, permiten observar que los ítems 5, 14 y 39 presentan pesos factoriales y comunalidad en el rango de valores aceptados; no obstante, se encuentran muy cercanos al límite inferior permitido. El valor bajo de la comunalidad indica que gran parte de la variabilidad del item no la están explicando los factores extraídos.

TABLA 3. Pesos factoriales sobre los cuatro factores extraídos

\begin{tabular}{|l|l|c|c|c|c|}
\hline \multirow{2}{*}{$\mathbf{N}^{\circ}$} & \multicolumn{1}{|c|}{ İtem } & \multicolumn{3}{|c|}{ Componente } \\
\cline { 3 - 5 } 1 & \multicolumn{1}{|c|}{$\begin{array}{l}\text { ¿Es difícil para usted soportar el } \\
\text { cansancio/agotamiento provocados } \\
\text { por su enfermedad? }\end{array}$} & 0,586 & 0,144 & 0,177 & $-0,021$ \\
\hline 2 & $\begin{array}{l}\text { ¿Son para usted molestos los cam- } \\
\text { bios de apetito? }\end{array}$ & 0,547 & 0,133 & 0,046 & $-0,150$ \\
\hline 3 & $\begin{array}{l}\text { ¿Es frecuente para usted sentir } \\
\text { dolor? }\end{array}$ & 0,423 & 0,099 & 0,153 & $-0,231$ \\
\hline 4 & $\begin{array}{l}\text { ¿Son incómodos para usted los } \\
\text { cambios en el sueño ocasionados } \\
\text { por la enfermedad? }\end{array}$ & 0,434 & 0,181 & 0,197 & $-0,168$ \\
\hline
\end{tabular}




\begin{tabular}{|c|c|c|c|c|c|}
\hline \multirow{2}{*}{$\mathbf{N} .^{\circ}$} & \multirow{2}{*}{ Ítem } & \multicolumn{4}{|c|}{ Componente } \\
\hline & & 1 & 2 & 3 & 4 \\
\hline 5 & $\begin{array}{l}\text { ¿Hasta qué punto es molesto para } \\
\text { usted el estreñimiento? }\end{array}$ & 0,353 & 0,054 & $-0,093$ & $-0,242$ \\
\hline 6 & $\begin{array}{l}\text { ¿Hasta qué punto es molesto para } \\
\text { usted sentir náuseas? }\end{array}$ & 0,488 & 0,123 & 0,117 & $-0,261$ \\
\hline 7 & $\begin{array}{l}\text { ¿La enfermedad ha afectado su } \\
\text { funcionamiento sexual? }\end{array}$ & 0,203 & 0,063 & 0,801 & $-0,018$ \\
\hline 8 & $\begin{array}{l}\text { ¿Cómo percibe su salud física en } \\
\text { general actualmente? }\end{array}$ & 0,591 & 0,014 & 0,153 & 0,045 \\
\hline 9 & $\begin{array}{l}\text { ¿Qué tan difícil ha sido manejar la } \\
\text { rutina diaria con su enfermedad? }\end{array}$ & 0,524 & 0,176 & 0,181 & 0,021 \\
\hline 10 & $\begin{array}{l}\text { ¿Cómo percibe su calidad de vida } \\
\text { actualmente? }\end{array}$ & 0,619 & 0,104 & 0,098 & 0,106 \\
\hline 11 & $\begin{array}{l}\text { ¿Qué tan feliz se siente actualmen- } \\
\text { te? }\end{array}$ & 0,637 & 0,196 & $-0,024$ & 0,215 \\
\hline 12 & $\begin{array}{l}\text { ¿Siente que tiene control sobre los } \\
\text { diferentes aspectos que afectan su } \\
\text { vida diaria? }\end{array}$ & 0,457 & 0,061 & $-0,195$ & 0,160 \\
\hline 13 & $\begin{array}{l}¿ Q u e ́ \text { tan satisfecho se siente con su } \\
\text { vida actualmente? }\end{array}$ & 0,612 & 0,074 & $-0,039$ & 0,279 \\
\hline 14 & $\begin{array}{l}\text { ¿Qué tan buena es su capacidad } \\
\text { para concentrarse y recordar cosas } \\
\text { actualmente? }\end{array}$ & 0,317 & 0,068 & $-0,301$ & $-0,103$ \\
\hline 15 & $\begin{array}{l}\text { ¿Qué tan útil se siente actualmen- } \\
\text { te? }\end{array}$ & 0,722 & $-0,115$ & $-0,057$ & 0,157 \\
\hline 16 & $\begin{array}{l}\text { ¿Percibe usted que su enfermedad o } \\
\text { tratamiento ha causado cambios en } \\
\text { su apariencia? }\end{array}$ & 0,402 & 0,271 & 0,309 & 0,075 \\
\hline 17 & $\begin{array}{l}\text { ¿Ha ocasionado su enfermedad } \\
\text { cambios en la forma de verse usted } \\
\text { como persona? }\end{array}$ & 0,471 & 0,333 & 0,293 & 0,074 \\
\hline 18 & $\begin{array}{l}\text { ¿Le generó angustia conocer el } \\
\text { diagnóstico inicial de su enferme- } \\
\text { dad? }\end{array}$ & 0,110 & 0,682 & 0,083 & $-0,095$ \\
\hline 19 & $\begin{array}{l}\text { ¿Le genera angustia el tratamiento } \\
\text { que está recibiendo actualmente? }\end{array}$ & 0,275 & 0,493 & $-0,045$ & $-0,069$ \\
\hline
\end{tabular}




\begin{tabular}{|c|c|c|c|c|c|}
\hline \multirow{2}{*}{$\mathbf{N} .^{\circ}$} & \multirow{2}{*}{ Ítem } & \multicolumn{4}{|c|}{ Componente } \\
\hline & & 1 & 2 & 3 & 4 \\
\hline 20 & $\begin{array}{l}\text { ¿Le genera angustia el tiempo que } \\
\text { transcurre sin medicación? }\end{array}$ & 0,198 & 0,414 & 0,021 & $-0,248$ \\
\hline 21 & $\begin{array}{l}\text { ¿Qué tan ansioso/desesperado se } \\
\text { encuentra actualmente? }\end{array}$ & 0,260 & 0,551 & $-0,005$ & $-0,050$ \\
\hline 22 & $\begin{array}{l}\text { ¿Con qué frecuencia se siente depri- } \\
\text { mido o decaído? }\end{array}$ & 0,500 & 0,405 & $-0,064$ & $-0,020$ \\
\hline 23 & $\begin{array}{l}\text { ¿Le atemoriza la idea de hacerse } \\
\text { nuevos exámenes diagnósticos? }\end{array}$ & 0,230 & 0,521 & $-0,074$ & $-0,050$ \\
\hline 24 & $\begin{array}{l}\text { ¿Siente usted temor de sufrir una } \\
\text { enfermedad adicional a la que ya } \\
\text { tiene? }\end{array}$ & $-0,066$ & 0,700 & 0,082 & $-0,019$ \\
\hline 25 & $\begin{array}{l}\text { ¿Teme usted que su enfermedad le } \\
\text { pueda ocasionar deterioro? }\end{array}$ & 0,046 & 0,599 & 0,308 & 0,184 \\
\hline 26 & $\begin{array}{l}\text { ¿Percibe usted que su situación } \\
\text { actual de salud está empeorando? }\end{array}$ & 0,459 & 0,351 & 0,160 & 0,262 \\
\hline 27 & $\begin{array}{l}\text { ¿Percibe que su familia está preocu- } \\
\text { pado por su enfermedad? }\end{array}$ & $-0,178$ & 0,045 & $-0,028$ & 0,420 \\
\hline 28 & $\begin{array}{l}\text { ¿Se siente usted apoyado por otros } \\
\text { para manejar su enfermedad? }\end{array}$ & 0,228 & $-0,019$ & $-0,166$ & 0,442 \\
\hline 29 & $\begin{array}{l}\text { ¿Su condición de salud actualmen- } \\
\text { te ha afectado negativamente sus } \\
\text { relaciones personales? }\end{array}$ & 0,563 & 0,224 & 0,188 & 0,184 \\
\hline 30 & $\begin{array}{l}\text { ¿Ha sido impactada/afectada su } \\
\text { sexualidad por su enfermedad? }\end{array}$ & 0,202 & 0,074 & 0,789 & $-0,066$ \\
\hline 31 & $\begin{array}{l}\text { ¿Hasta qué punto ha interferido su } \\
\text { enfermedad y tratamiento con su } \\
\text { empleo? }\end{array}$ & 0,222 & 0,070 & 0,559 & $-0,136$ \\
\hline 32 & $\begin{array}{l}\text { ¿Percibe que su enfermedad y } \\
\text { tratamiento han interferido con sus } \\
\text { actividades/quehaceres en el hogar? }\end{array}$ & 0,607 & 0,221 & 0,195 & $-0,033$ \\
\hline 33 & $\begin{array}{l}\text { ¿Se siente usted aislado por motivo } \\
\text { de su enfermedad y tratamiento? }\end{array}$ & 0,552 & 0,217 & 0,151 & 0,016 \\
\hline 34 & $\begin{array}{l}\text { ¿Para usted son costosos su enfer- } \\
\text { medad y tratamiento? }\end{array}$ & 0,448 & 0,176 & 0,127 & $-0,113$ \\
\hline
\end{tabular}




\begin{tabular}{|l|l|l|l|l|l|}
\hline \multirow{2}{*}{$\mathbf{N}^{\circ}$} & \multicolumn{1}{|c|}{ Ítem } & \multicolumn{3}{c|}{ Componente } \\
\cline { 3 - 6 } 35 & $\begin{array}{l}\text { ¿Es para usted importante partici- } \\
\text { par en actividades religiosas como } \\
\text { hacer oración, ir a la iglesia o al } \\
\text { templo? }\end{array}$ & $-0,040$ & $-0,040$ & 0,062 & 0,413 \\
\hline 36 & $\begin{array}{l}\text { ¿Es importante para usted tener un } \\
\text { espacio de reflexión diaria? }\end{array}$ & 0,135 & $-0,152$ & 0,102 & 0,412 \\
\hline 37 & $\begin{array}{l}\text { ¿Considera usted que su condición } \\
\text { de enfermedad lo ha transformado } \\
\text { espiritualmente? }\end{array}$ & $-0,162$ & $-0,180$ & 0,060 & 0,567 \\
\hline 38 & $\begin{array}{l}\text { ¿Actualmente ve su futuro como } \\
\text { incierto? }\end{array}$ & 0,491 & 0,274 & 0,200 & 0,302 \\
\hline 39 & $\begin{array}{l}\text { ¿Asocia usted su enfermedad con } \\
\text { cambios positivos en su vida? }\end{array}$ & $-0,109$ & $-0,048$ & 0,132 & $-0,397$ \\
\hline 40 & $\begin{array}{l}\text { ¿Le encuentra sentido actualmente } \\
\text { a su vida? }\end{array}$ & 0,586 & 0,065 & $-0,212$ & 0,419 \\
\hline 41 & $\begin{array}{l}\text { ¿Siente esperanza/expectativa en el } \\
\text { manejo de su condición actual? }\end{array}$ & 0,300 & 0,054 & $-0,093$ & 0,543 \\
\hline
\end{tabular}

Fuente: elaboración propia

Al analizar la carga factorial de los items en los 4 factores extraídos e intentar establecer una relación con la estructuración de las dimensiones planteadas por el autor, no se encontró una coincidencia total entre estos 2 aspectos. Solo 22 de los 41 items son explicados por el factor subyacente a la dimensión planteada por el autor. Se observa, de este modo, una marcada discrepancia en las dimensiones de bienestar psicológico y bienestar social.

El factor 1 se identificó con la dimensión de bienestar físico. Según lo detallado en la tabla 3 , el ítem 7 no se vincula con este factor. Sin embargo, muestra una carga factorial de 0,81 . Por otro lado, muchos de los items pertenecientes por construcción a la dimensión psicológica (ítems 9 al 26) presentan una carga importante sobre el factor 1; el mismo comportamiento se observa en algunos ítems de la dimensión social (ítems 27 al 34), y 2 ítems de la dimensión espiritual (items 35 al 41) también presentan una carga factorial sobre el factor 1 . Teniendo en cuenta lo anterior, se concluye que las dimensiones de bienestar psicológico y bienestar social no están bien definidas.

El factor 2 puede identificarse con una parte de la dimensión de bienestar psicológico. Los ítems 18 al 25 se explican entre el 34 y $50 \%$ por 142 este factor. A diferencia de estos, los ítems del 9 al 17 y el 26 parecen ser mejor explicados por el factor 1 . El item 24, por su parte, resulta tener mayor 
carga factorial en este factor, lo que no sorprende, pues este evalúa el temor de tener una enfermedad adicional a la enfermedad crónica que ya padece, aspecto muy afín con el bienestar psicológico.

El factor 3 puede identificarse con dos ítems del bienestar social y uno de la dimensión de bienestar físico. Los ítems 7 y 30 se explican en una proporción importante por este factor. Los ítems ubicados en la dimensión de bienestar social por los autores se encuentran explicados en su mayoría por el factor 1 y 4 así: factor 1 (29, 32, 33 y 34), factor 4 (27 y 28); por lo tanto, el factor 3 no se vincula con la dimensión de bienestar social; esta dimensión no es precisa en el análisis realizado.

El factor 4 se identifica con la dimensión de bienestar espiritual. Los items 37 y 41 registran porcentajes superiores al $50 \%$ por este factor. El ítem 39 presenta una carga factorial cercana al limite inferior y se identifica como un item propenso a ser eliminado.

Aunque el número de factores resultante de la solución del análisis factorial exploratorio pudo aproximarse al número de dimensiones planteado por los autores, las cargas factoriales de 19 items no corresponden exactamente con los ítems diseñados y planteados por los autores para medir cada dimensión. Se observa especial discrepancia en las dimensiones psicológica y social. La ausencia de la segunda de estas dos condiciones no permite que se tenga evidencia de la validez de constructo del instrumento en las 4 dimensiones propuestas inicialmente (22).

Lo anterior plantea la necesidad de encontrar una explicación al comportamiento observado para los ítems 7, 9-17, 26-29, 32-34, 38 y 40. El análisis de la validez de constructo utilizando técnicas de análisis factorial confirmatorio permitiría estimar el aporte de un item a una o varias dimensiones simultáneamente, así como la determinación de posibles correlaciones existentes entre las dimensiones.

\section{Confiabilidad}

El coeficiente alfa de Cronbach calculado con los datos de la investigación fue de 0,88. Según George y Mallery, citados por Carvajal y cols. (23), el alfa de Cronbach por debajo de 0,5 muestra un nivel de fiabilidad no aceptable. De 0,5 a 0,6 se podría considerar un nivel pobre; si se situara entre 0,7 y 0,8 haria referencia a un nivel aceptable; en el intervalo 0,8-0,9 se podría calificar como de un nivel bueno, y si tomara un valor superior a 0,9, sería excelente. La confiabilidad del Instrumento Calidad de Vida de Betty Ferrell para Personas con Enfermedad Crónica reporta una consistencia interna buena.

A pesar de lo anterior, la remoción de algunos ítems deja ver un leve incremento del coeficiente. La curva de Mesbah mostrada en la figura 1 presenta el valor para el coeficiente alfa de Cronbach al realizar la remoción gradual de los ítems. Se observa que al ser eliminados los ítems 27, 35, 37 y 39, el coeficiente alcanza su máximo en valores alrededor de 0,887 .

Puede observarse que al remover los ítems 27, 35, 37 y 39, el coeficiente alfa incrementa, lo cual indica que estos items afectan negativamente la confiabilidad del instrumento. 


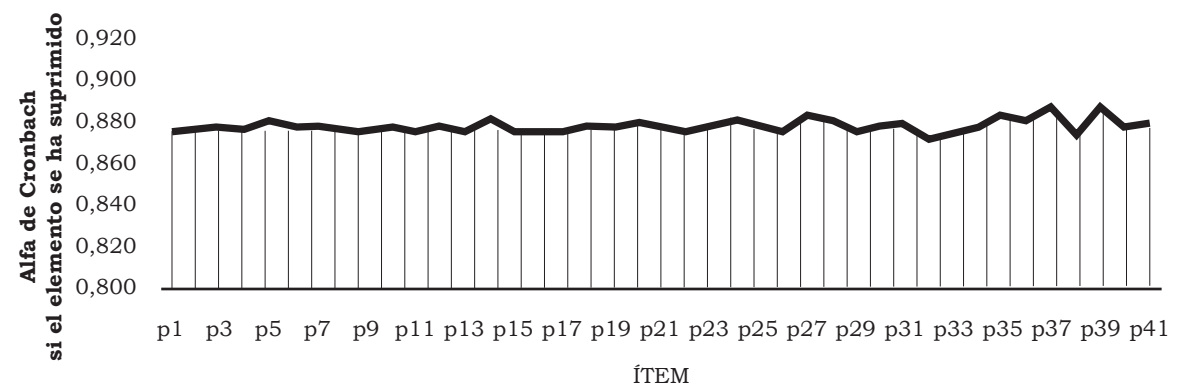

Figura 1. Curva de Cronbach-Mesbah con los 41 items

Fuente: elaboración propia

\section{Correlación entre ítems}

Se revisó la correlación entre ítems para considerar la eliminación de ítems altamente correlacionados. El par de ítems 7 y 30 mostraron una correlación fuerte de 0,82; las dos preguntas se refieren a la sexualidad, por lo tanto, se debe considerar la remoción de alguno de los ítems y dejar solo uno que sintetice adecuadamente el tema de sexualidad en las personas con enfermedad crónica.

\section{Discusión}

Es de anotar que este es el primer ejercicio formal y sistemático realizado con el fin de determinar la validez y la confiabilidad del instrumento de calidad de vida de personas con enfermedad crónica propuesto por Betty Ferrell, por lo que no existen estudios con los cuales hacer una comparación detallada.

En relación con la validez de contenido, en el presente estudio los ítems 10 y 20 no cumplieron con el índice mínimo establecido para considerarlos esenciales por los expertos en la medición de la calidad de vida. Es de anotar que el ítem 10 es una pregunta global respecto a cómo se percibe la calidad de vida actualmente, aspecto que para varios expertos puede ser redundante. El ítem 20 indaga "si se genera angustia en el tiempo que transcurre sin medicación", situación poco usual en los enfermos crónicos, teniendo en cuenta que una de sus principales problemáticas es la polifarmacia y la necesidad de medicamentos permanente.

En cuanto a la validez de constructo, estos items presentaron un adecuado peso factorial y comunalidad; sin embargo, el ítem 39 estuvo muy cercano al límite inferior permitido, lo que cuestionaría la permanencia de este en próximas aplicaciones del instrumento. Los ítems 10, 12, 29 y 40 no se relacionaron con las dimensiones planteadas por los autores del instrumento; sin embargo, estos hallazgos se deben contrastar a partir de lo reportado en la validez de contenido, ya que fueron considerados eseencia144 les para medir la calidad de vida de personas con enfermedad crónica por parte de los expertos. 
Por otro lado, teniendo en cuenta que aun cuando existen varios instrumentos que miden la calidad de vida y que las escalas Medical OutcomeStudy Short Form (MOSSF-36) (24) y el World Health Organization Quality of Life (WHOQOL-BREF) (24), propuesto por la Organización Mundial de la Salud, son las de mayor uso reportado por la literatura, cuentan con procesos de validez y confiabilidad para Colombia, se considera pertinente contrastar los resultados obtenidos en la presente investigación, respecto a estas escalas de referencia.

El MOSSF-36 es un instrumento genérico de medición que consta de 36 items que se agrupan en 7 dominios: dolor corporal, desempeño emocional, desempeño físico, función física, salud general, salud mental y vitalidad. Las pruebas psicométricas realizadas en Colombia por Cardona, Ospina y Eljadue (24) reportan una fiabilidad evaluada a través del alfa de Cronbach >0,70; lo mismo reportan Lugo, García y Gómez: un alfa de Cronbach >0,70 (25). La consistencia interna se evaluó a partir del rango de los coeficientes de correlación de Pearson de los puntos con el dominio al cual pertenecen. Cardona, Ospina y Eljadue reportan coeficientes de correlación entre los dominios del MOSSFF-36, que fueron $>0,40$, exceptuando la correlación entre el desempeño emocional y la función física. Entre tanto, Lugo, García y Gómez reportan una correlación ítem escala $>0,48$. Al ser comparado con la consistencia interna realizada al Instrumento Calidad de Vida de Betty Ferrell para Personas con Enfermedad Crónica, se evidencia que los instrumentos cuentan con una confiabilidad adecuada; pero discrepan los indices de correlación, pues para el instrumento de la presente investigación no todos los índices item-escala son mayores de 0,4.

Es de anotar que el instrumento MOSSFF-36 hace hincapié en la funcionalidad y la dependencia de la persona a través de la agrupación en los 8 dominios descritos. Aunque el instrumento de calidad de vida de Ferrell incluye ítems relacionados con los dominios de la MOSSFF-36, su propósito va más allá de la funcionalidad, pues el relieve está en las dimensiones psicológica, social y espiritual. De acuerdo con los hallazgos, los items de la dimensión espiritual reportaron mayor relación con el constructo propuesto por los autores, lo que hace suponer la importancia de este componente en la percepción de la calidad de vida de personas con enfermedad crónica.

Por otro lado, al revisar los pesos factoriales de los items 1 a 17 del Instrumento Calidad de Vida de Betty Ferrell, que se agrupan en un solo factor, se relacionan estrechamente con los dominios función física, salud general, salud mental y vitalidad de la escala MOSSFF-36, hallazgo que hace suponer la importancia de realizar pruebas psicométricas de validez de criterio de las dos escalas en estudios posteriores.

El WHOQOL-BREF es un instrumento genérico derivado del WHOQOL-100, y consta de 26 ítems, 24 de ellos se agrupan en 4 dominios: salud física, salud psicológica, ambiente y relaciones sociales, y los 2 restantes cualifican la calidad de vida y la salud. Se le han realizado pruebas psicométricas en el contexto local que reportan una confiabilidad por medio del alfa de Cronbach $>0,70$ y validez discriminante a través del coeficiente de correlación de Pearson entre preguntas y dimensiones del instrumento, las cuales fueron $>0,40$. El 
dominio con el peor resultado se obtuvo en las relaciones sociales en el instrumento WHOQOL-BREF, hallazgo que coincide con lo identificado en la validez de constructo de la dimensión social correspondiente al Instrumento de Calidad de Vida de Betty Ferrell de Personas con Enfermedad Crónica, al no obtenerse equivalencia con las propuestas por los autores. Estos resultados implican ahondar en los componentes de relaciones sociales e interacción referidos y percibidos por los participante.

Es importante aclarar que las pruebas psicométricas realizadas a los instrumentos MOSSFF-36 y WHOQOL-BREF no fueron las mismas realizadas al Instrumento de Calidad de Vida de Betty Ferrell para Personas con Enfermedad Crónica, lo que hace que la comparación de los resultados entre dimensiones sea limitada.

\section{Conclusiones}

El Instrumento Calidad de Vida de Betty Ferrell para Personas con Enfermedad Crónica en español reporta suficiencia, coherencia y relevancia a partir del índice de kappa y un alto índice de validez de contenido.

En el análisis de ítems, la evaluación conjunta de validez y confiabilidad permitió en este estudio definir criterios para eliminar o conservar items sin favorecer exclusivamente la confiabilidad del instrumento y sin poner en riesgo su validez de constructo y de contenido. La falta de evidencia de validez de constructo encontrada por el método de análisis factorial exploratorio utilizado plantea la necesidad de encontrar una explicación al comportamiento observado por los items 9-17, 26-29, 32-34, 39 y 40, por lo se considera la necesidad de realizar un análisis factorial confirmatorio en próximas investigaciones.

\section{Limitaciones}

La poca disponibilidad de información en estudios sobre calidad de vida que han utilizado el mismo instrumento que indiquen sus propiedades psicométricas, lo que limitó ampliar la discusión.

\section{Conflicto de intereses}

Las investigadoras no presentan conflictos de intereses.

\section{Financiación}

Programa para Disminuir la Carga de la Enfermedad Crónica en Colombia. Convocatoria 537- 2010 Colciencias. Unión Temporal Carga de la Enfermedad Crónica en Colombia.

\section{Referencias}

1. World Health Organization (WHO). Global Status Report on noncommunicable

2. furnaleto C, Carmona M. A inluência da síndrome metabólica sobre a qualidade de vida de pacientes em Sao Caetano do Sul. Rev Soc Bras. 2009;34(2):85-99. 
3. Organización Mundial de la Salud (OMS). Calidad de vida. En: Programas nacionales de lucha contra el cáncer: directrices sobre política y gestión. Ginebra: OMS; 2002. p. 84-6.

4. Oblitas L. Psicología de la salud y enfermedades crónicas. Bogotá: PSICOM; 2006.

5. Ferrell B, Dow H, Grant M. Measurement of the quality of life in cancer survivors. Qual Life Res. 1995;4(6):523-31.

6. Velarde E, Ávila C. Consideraciones metodológicas para evaluar la calidad de vida. Salud Pública Méx. 2002;44(5):448-63.

7. Nunnally J, Bernestein H. Teoría psicométrica. 3a ed. México: McGrawHill; 1995.

8. Tristán A. Modificación de los modelos de Lawshe para el dictamen cuantitativo de la validez de contenido de un instrumento objetivo. Avances en Medición. 2006;6:37-48.

9. Herrans L. Psicología y medición: el desarrollo de las pruebas psicológicas en Puerto Rico. 2a ed. México: McGraw-Hill Interamericana; 2000.

10. Fleiss J. Measuring nominal scale agreement among many raters. Psycological Bulletin. 1971;76(5):378-82.

11. Escobar J, Cuervo A. Validez de contenido y juicio de expertos: una aproximación a su utilización. Avances de Medición. 2008;6:27-36.

12. Argibay J. Muestra en investigación cuantitativa. Subj Procesos Cogn. 2009;13(1):13-29.

13. Lévy J, Varela J. Modelización de covarianzas en ciencias sociales. Madrid: Gesgiblo; 2006.

14. Norman G, Striner D, Tarrés J. Bioestadística. Madrid: Mosby/Doyma; 1996.

15. Grupo al Paciente Crónico y su Familia. Instrumento de caracterización del paciente con enfermedad crónica Versión 3, 2013. Bogotá: Universidad Nacional de Colombia; 1996-2012.

16. Mesbah M. Statistical quality of life. En: Balakrishnan N, editor. Method an aplications of statistics in the life and health sciences. Hoboken, NJ: Wiley; 2010. p. 839-64.

17. Resolución 008430/1993, por la cual se establecen las normas científicas, técnicas y administrativas para la investigación en salud. República de Colombia, Ministerio de Salud.

18. Ley $911 / 2004$, por la cual se dictan disposiciones en materia de responsabilidad deontológica para el ejercicio de la profesión de enfermería en Colombia (Diario Oficial 45.693, de 06-10-2004).

19. Consejo de Organizaciones Internacionales de las Ciencias Médicas. Pautas éticas internacionales para la investigación biomédica en seres humanos preparadas por el Consejo de Organizaciones Internacionales de las Ciencias Médicas (CIOMS). Ginebra; 2012.

20. Universidad Nacional de Colombia. Consejo Superior Universitario. Política Ambiental de la Universidad Nacional de Colombia; 2011.

21. Raykov T, Marcoulides G. An introduction to applied multivariate analysis. New York: Routledge; 2008. 
22. Waltz C, Strikland O, Lenz E. Measurement in nursing and health research. 4a ed. New York: Springer; 2010.

23. Carvajal A, Centeno C, Watson R, Martínez M, Rubiales A. ¿How is an instrument for measuring health to be validated? Anales del Sistema Sanitario de Navarra. 2011;34(1):63-72.

24. Cardona J, Ospina L, Eljadue A. Validez discriminante, convergente/ divergente, fiabilidad y consistencia interna, del WHOQOL-BREF y el MOSSF-36 en adultos sanos de un municipio colombiano. Colomb Med. 2009;42:438-47.

25. Lugo L, García H, Gómez C. Confiabilidad del cuestionario de calidad de vida en salud SF-36 en Medellín, Colombia. Rev Fac Nac Salud Pública. 2015;33(1):50-7. 